\title{
Status of the GEO600 detector
}

H Lück ${ }^{1}$, M Hewitson ${ }^{1}$, P Ajith ${ }^{1}$, B Allen ${ }^{3}$, P Aufmuth ${ }^{1}$, C Aulbert $^{3}$, S Babak ${ }^{3}$, R Balasubramanian ${ }^{4}$, B W Barr ${ }^{2}$, S Berukoff $^{3}$, A Bunkowski $^{1}$, G Cagnoli ${ }^{2}$, C A Cantley ${ }^{2}$, M M Casey ${ }^{2}, \mathrm{~S} \mathrm{Chelkowski}^{1}, \mathrm{Y} \mathrm{Chen}^{3}$, D Churches $^{4}$, T Cokelaer $^{4}$, C N Colacino ${ }^{6}$, D R M Crooks ${ }^{2}$, C Cutler $^{3}$, K Danzmann 1 , R J Dupuis ${ }^{2}$, E Elliffe ${ }^{2}$, C Fallnich ${ }^{5}$, A Franzen ${ }^{1}$, A Freise ${ }^{6}$, I Gholami $^{3}$, S Goßler ${ }^{1}$, A Grant ${ }^{2}$, H Grote ${ }^{1}$, S Grunewald ${ }^{3}$, J Harms ${ }^{1}$,

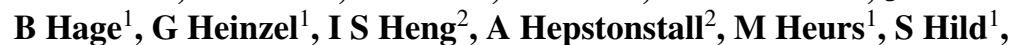
J Hough ${ }^{2}$, Y Itoh ${ }^{3}$, G Jones ${ }^{4}$, R Jones ${ }^{2}$, S H Huttner ${ }^{2}$, K Kötter ${ }^{1}$, B Krishnan ${ }^{3}$, P Kwee ${ }^{1}$, M Luna ${ }^{7}$, B Machenschalk ${ }^{3}$, M Malec ${ }^{1}$, R A Mercer ${ }^{6}$, T Meier ${ }^{1}$, C Messenger ${ }^{6}$, S Mohanty ${ }^{3}$, K Mossavi $^{1}$, S Mukherjee ${ }^{3}$, P Murray ${ }^{2}$, G P Newton ${ }^{2}$, M A Papa ${ }^{3}$, M Perreur-Lloyd ${ }^{2}$, M Pitkin ${ }^{2}$, M V Plissi ${ }^{2}, \mathbf{R}$ Prix $^{3}$, V Quetschke ${ }^{1}$, V Re $^{6}$, T Regimbau $^{4}$, H Rehbein ${ }^{1}$, S Reid ${ }^{2}$, L Ribichini ${ }^{1}$, D I Robertson ${ }^{2}$, N A Robertson ${ }^{2,8}$, C Robinson ${ }^{4}$, J D Romano ${ }^{4}$, S Rowan ${ }^{2}$, A Rüdiger ${ }^{1}$, B S Sathyaprakash ${ }^{4}$, R Schilling ${ }^{1}$, R Schnabel ${ }^{1}$, B F Schutz ${ }^{3,4}$, F Seifert ${ }^{1}$, A M Sintes ${ }^{7}$, J R Smith ${ }^{1}$, P H Sneddon ${ }^{2}$, K A Strain ${ }^{2}$, I Taylor ${ }^{4}$, R Taylor ${ }^{2}$, A Thüring ${ }^{1}$, C Ungarelli ${ }^{6}, \mathbf{H}$ Vahlbruch ${ }^{1}, \mathbf{A}$ Vecchio $^{6}, \mathrm{~J} \mathrm{Veitch}^{2}, \mathbf{H}$ Ward $^{2}, \mathbf{U}$ Weiland $^{1}$, H Welling ${ }^{5}$, L Wen ${ }^{3}$, P Williams ${ }^{3}$, B Willke ${ }^{1}$, W Winkler ${ }^{1}$, G Woan ${ }^{2}$ and $\mathbf{R} \mathbf{Z h u}^{3}$

\footnotetext{
${ }^{1}$ Zentrum für Gravitationsphysik, Callinstr. 38, D-30167 Hannover, Germany

2 Department of Physics \& Astronomy, University of Glasgow, Glasgow G12 8QQ, UK

${ }^{3}$ Max-Planck-Institut für Gravitationsphysik (Albert-Einstein-Institut), Golm, Am Mühlenberg 1, D-14476 Golm, Germany

${ }^{4}$ Department of Physics and Astronomy, Cardiff University, PO Box 913, Cardiff CF2 3YB, UK

${ }^{5}$ Laser Zentrum Hannover e. V., Hollerithallee 8, D-30419 Hannover, Germany

${ }^{6}$ School of Physics and Astronomy, The University of Birmingham, Edgbaston, Birmingham B15 2TT, UK

${ }^{7}$ Departament de Fisica, Universitat de les Illes Balears, E-07122 Palma de Mallorca, Spain

${ }^{8}$ Edward L Ginzton Laboratory, Stanford University, Stanford, CA 94305-4088, USA

E-mail: harald.lueck@aei.mpg.de
}

Received 7 September 2005, in final form 29 November 2005

Published 24 March 2006

Online at stacks.iop.org/CQG/23/S71

\begin{abstract}
Of all the large interferometric gravitational-wave detectors, the German/British project GEO600 is the only one which uses dual recycling. During the four weeks of the international S4 data-taking run it reached an instrumental duty cycle of $97 \%$ with a peak sensitivity of $7 \times 10^{-22} \mathrm{~Hz}^{-1 / 2}$ at $1 \mathrm{kHz}$. This paper describes the status during S4 and improvements thereafter.
\end{abstract}


PACS numbers: $04.80 . \mathrm{Nn}, 95.55 . \mathrm{Ym}$

(Some figures in this article are in colour only in the electronic version)

\section{Introduction}

The gravitational-wave detector GEO600 [1] is on the way to design sensitivity (see figure 1 and [2]). For the LSC (LIGO Scientific Collaboration) wide data-taking run S4 (4th scientific data-taking run, 22 February to 23 March 2005) the sensitivity of the GEO600 detector was optimized in the $1 \mathrm{kHz}$ range. This frequency was a compromise between best sensitivity for coalescing binary systems, which calls for low frequency sensitivity, and best peak sensitivity, which in the GEO600 case during S4 favoured high frequencies.

\section{Overview}

GEO600 is an interferometric gravitational-wave detector built and operated by a British/German collaboration in northern Germany close to Hannover. Although GEO600 is smaller than the other large-scale interferometric GW-detectors in the US $(2 \mathrm{~km}$ and $4 \mathrm{~km}$ arm length) and Italy (3 km arm length) the sensitivity aimed for is roughly the same. To compensate for the shorter arm length, GEO600 incorporates advanced techniques that are partly foreseen for the later stages of the larger detectors, e.g. signal recycling, glass fibre suspensions and multiple stage pendulums. While the LIGO detectors have essentially reached their design sensitivity, GEO600 and VIRGO still need to improve by about an order of magnitude.

GEO600 is operated as a dual recycled Michelson interferometer [3] with folded $600 \mathrm{~m}$ arms, yielding an effective arm length of $1200 \mathrm{~m}$ (see figure 2). Currently, $5 \mathrm{~W}$ from the light of a $12 \mathrm{~W}$ master/slave laser system [4] is spatially filtered by two successive triangular mode cleaners (both having slightly different round trip lengths of about $8 \mathrm{~m}$ ) and then sent into the power recycling (PR) cavity. The optical losses inside the mode cleaners and the other optical components in the beam path give a power of $1.7 \mathrm{~W}$ at the power recycling mirror (MPR). In the final set-up the full $12 \mathrm{~W}$ from the laser will give a power of about $5 \mathrm{~W}$ incident upon the interferometer. Schnupp phase modulation for the longitudinal control of the Michelson interferometer and of the signal recycling (SR) cavity is applied between the second mode cleaner and the PR cavity. Control signals are picked up at the output port and from a parasitic reflex of the anti-reflection coating of the beam splitter (BS) for the Michelson and the SR control, respectively. Where a quiet actuation reference is required, i.e. at the inboard mirrors (MCe and MCn), the signal recycling mirror (MSR) and MPR [5], the interferometer mirrors are suspended as triple pendulums with an identical reaction pendulum next to it. The lowest stages of the main interferometer mirrors are monolithically suspended [6].

\subsection{Dual recycling}

In contrast to the other large interferometers, GEO600 does not use arm cavities to enhance the light power inside the interferometer arms but instead relies on a high PR factor. In the final configuration, the light power of about $5 \mathrm{~W}$ being transmitted through the mode cleaners is enhanced up to $10 \mathrm{~kW}$ inside the PR cavity. In the following, the power inside the PR cavity, i.e. between MPR and BS, will be called PR power. 

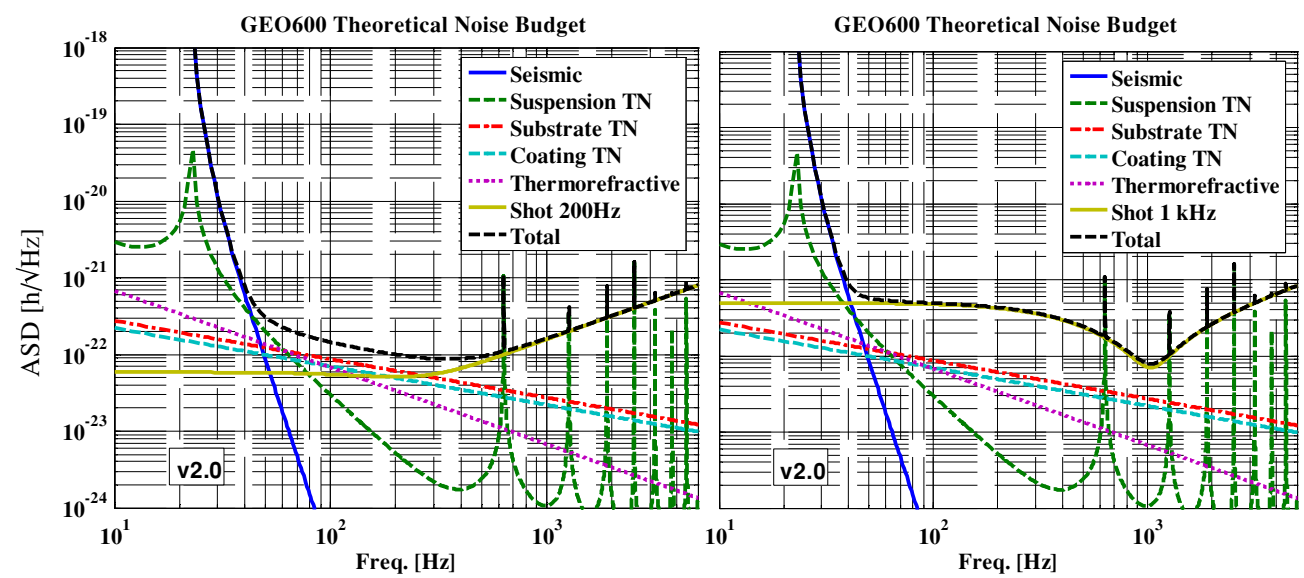

Figure 1. Noise contributions to the design sensitivity of GEO600. The shot noise contribution is shown for a power inside the PR cavity of $7 \mathrm{~kW}$ and for tunings of the signal recycling cavity of $1 \mathrm{kHz}$ and $200 \mathrm{~Hz}$ at the right- and left-hand side, respectively.

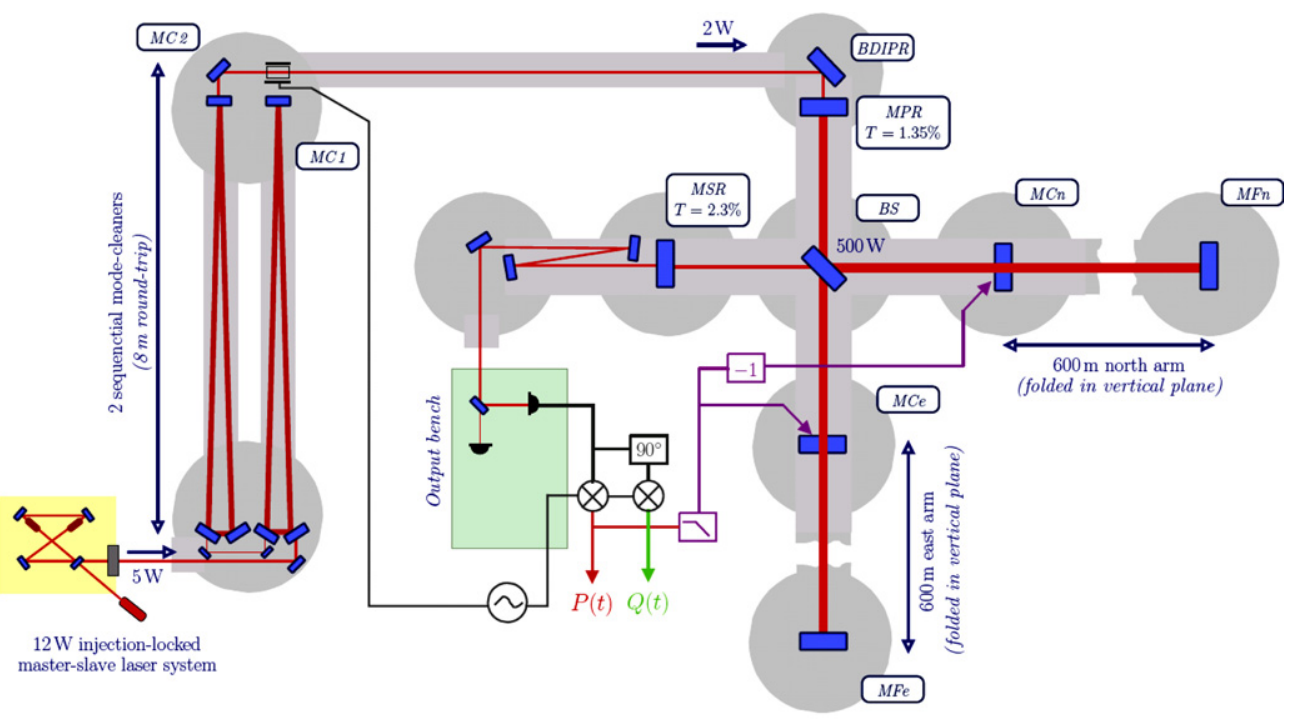

Figure 2. Optical layout of GEO600. In the set-up during the $\mathrm{S} 4$ data-taking run the powerand the signal recycling mirrors had a transmission of $1.35 \%$ and about $2 \%$, respectively. The interferometer arms are folded in the vertical plane giving an effective arm length of $1200 \mathrm{~m}$. The figure also includes a highly simplified diagram of the differential-Michelson-length control servo.

The PR power that can be obtained depends on the reflectivity of MPR and the intra-cavity losses. At high PR powers a thermal lens forming inside BS leads to different wave-front curvatures and beam radii of the two beams returning from the interferometer arms. The losses caused by the resulting decreased interferometer contrast dominate the overall cavity losses and thus limit the power build-up in the PR cavity. With a design value of $1000 \mathrm{ppm}$ for the transmission of MPR, the theoretical limit of the PR enhancement factor is $4000(=4 / 1000 \mathrm{ppm})$ if the intra-cavity losses are negligible. The assumed thermal lensing in BS leads to the value of 2000 mentioned above. 
Signal recycling (SR) allows the selection of bandwidth and the frequency of maximum sensitivity of a dual recycled detector. The bandwidth of the SR cavity for GEO600 with a transmission of MSR around $2 \%$ is about $400 \mathrm{~Hz}$. In GEO600 the minimum of the sensitivity curve can be tuned from $200 \mathrm{~Hz}$ to $5 \mathrm{kHz}$.

\subsection{Auto-alignment}

In order to achieve the required sensitivity of the Michelson interferometer, the axes of interfering beams have to be superimposed with a residual angle of about $10^{-8} \mathrm{rad}$. The beam spots have to be centred on the mirrors to minimize coupling of alignment noise into longitudinal signals. GEO600 is equipped with an automated alignment system that controls all relevant degrees of freedom [7]. This automation allowed locking times of more than $121 \mathrm{~h}$ to be achieved.

\section{Calibration}

In a dual recycled interferometer, the shape of the sensitivity curve not only depends on the bandwidth of the signal recycling cavity but also on the tuning, i.e. the microscopic position of the signal recycling mirror. Hence not only the magnitude of the interferometer response to gravitational waves needs to be calibrated but also the centering and the width of the resonance.

For continuous calibration, a set of calibration lines (see figure 4) is fed to the inboard mirrors via the electrostatic actuators. The amplitudes of the calibration lines give sufficient SNR to obtain a calibration rate of $1 \mathrm{~Hz}$ [8]. In a detuned dual recycled interferometer the asymmetry of the control and signal sidebands with respect to the cavity resonance gives an output RF signal that contains gravitational wave information in both demodulation quadratures $\mathrm{P}$ and $\mathrm{Q}$ (see figure 2). Due to the dispersion of the signal recycling cavity, the content in each demodulation quadrature depends on the signal frequency under consideration. To extract the maximum SNR from the interferometer output, both quadratures of the RF signal are demodulated and combined using optimized filter functions [9].

In order to have an independent check on the calibration of the electrostatic actuators, photon pressure actuation was used. The light of an amplitude-modulated fibre-coupled laser diode with a modulated power of about $1 \mathrm{~W}$ was collimated and reflected off the back of the far north (folding) mirror (MFn). Figure 3 shows the frequency dependency of the interferometer response. The behaviour follows a $1 / \mathrm{f}^{2}$ law and the calibration constant agrees well with the one obtained from the electrostatic actuators.

\section{Automation}

Although most control circuits of GEO600 are done in analogue electronics, digital interfaces allow computer control of many parameters. About ten PCs running LabVIEW ${ }^{\mathrm{TM}}$ Software are dedicated for manual and automated control of mirror pointing, setting and reading parameters for analogue control servos, tuning of the SR cavity, etc. This computer control allows fast recovery times in the event of a lock loss, i.e. the deviation of the interferometer from its operation point beyond the capture range of the servos. Complete computer control also allows operating the detector without human interaction overnight or at weekends. A feedforward system minimizing the longitudinal motion of the Michelson mirrors and reducing the tilt of the intermediate pendulum mass is implemented with a digital servo loop [10]. 


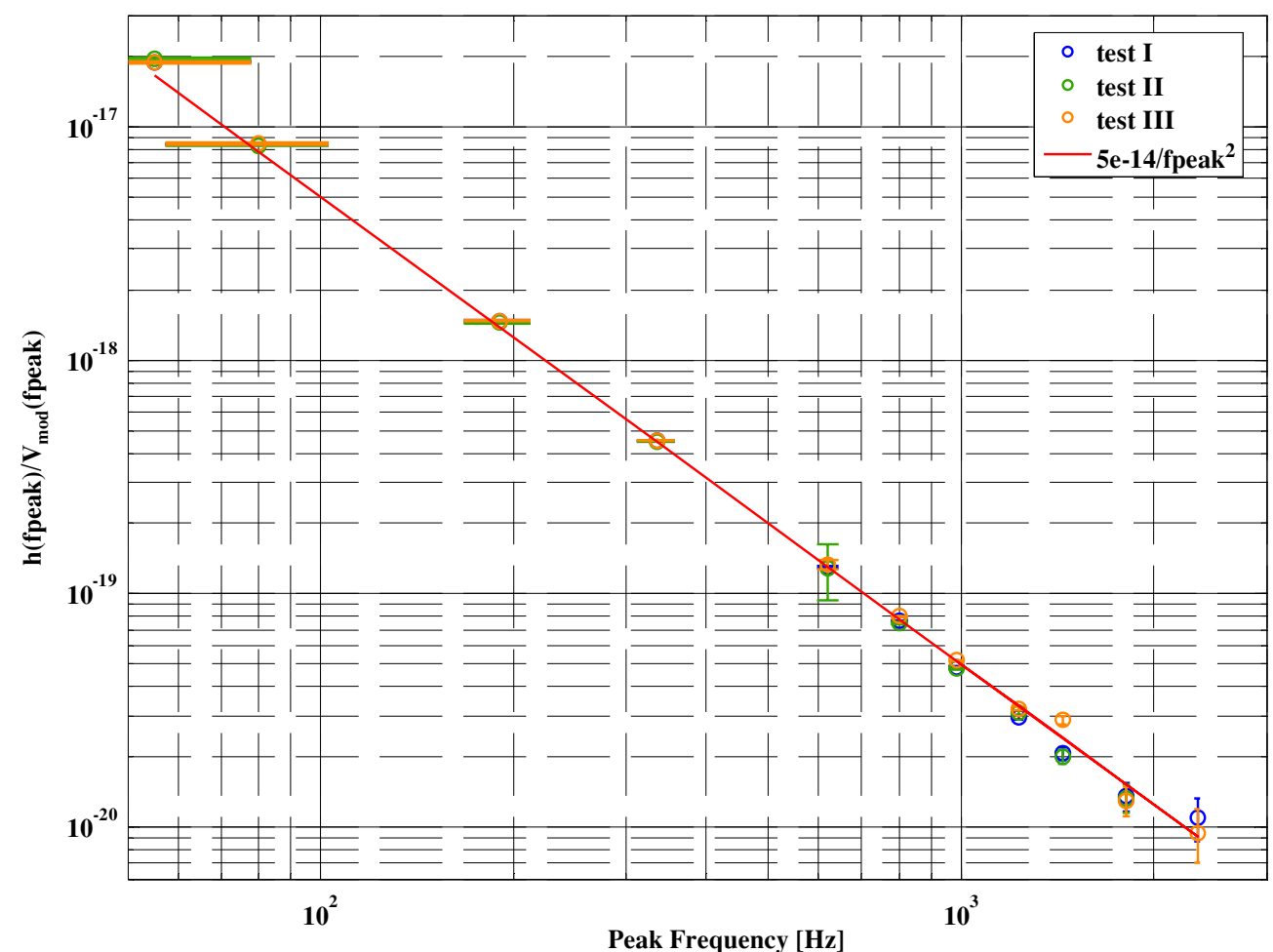

Figure 3. Interferometer response (measured line peak heights/applied modulation sine wave voltages) to signal applied to the radiation pressure actuator for different positions on the far north mirror.

\section{S4 status and performance}

During the S4 data run GEO600 was operated with a transmission of MPR of $1.35 \%$ yielding a PR power of about $500 \mathrm{~W}$. The transmission of MSR was about $2 \%$ as stated above. A typical sensitivity curve during S4 is shown in figure 4. Above $500 \mathrm{~Hz}$ the uncalibrated noise power spectrum of the Michelson output is frequency independant and hence the shape of the sensitivity curve above $500 \mathrm{~Hz}$ is given by the resonance of the SR cavity. The instrumental duty cycle, i.e. the fraction of time the detector was held at its operating point and was taking data, during S4 was 96.5\%. During the whole S4 run GEO600 was operated in a fully automated mode with at least one operator on site in two daily shifts from 5 to 21 UTC. An 'expert on duty' was on call all round the clock to be alerted in case of a problem either by the operator or out of shift hours by SMS alarms sent by the LabVIEW ${ }^{\mathrm{TM}}$ system.

Within the first nine days of S4, GEO600 showed extended periods of enhanced noise which eventually could be tracked down to a suspended optics platform (MU3, which carries Faraday isolators and electro-optical modulators) touching the support structure. This caused glitches in the Michelson output during seismically quiet times when enough strain could build up in the touching spot to generate macroscopic jumps, comparable to earthquakes that happen when the built-up strain gets high enough to overcome some threshold. These events resulted in a series of short large spikes in the Michelson differential error point signals. After rotating MU3 it no longer touched the suspension frame and the excess noise was gone. Taking out the disturbed sections from the detector data still gives a duty cycle above 95\%. Even if we 


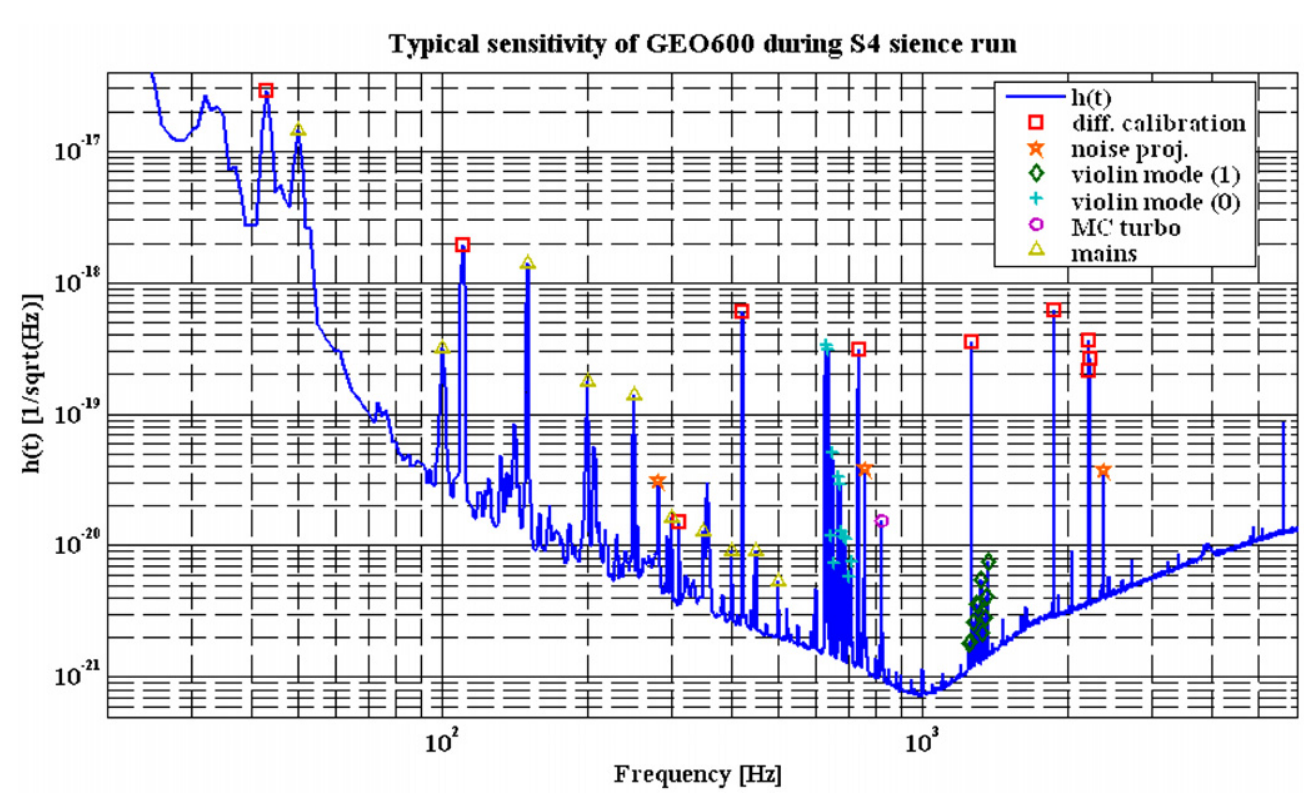

Figure 4. Sensitivity of GEO600 during the S4 data run. The marks show identified lines in the spectrum which mostly are calibration lines (red (in the online version) squares), lines injected for monitoring the constancy of noise projection transfer functions (orange stars) and violin modes. The blue plus signs indicate the fundamental violin modes whereas the green diamonds indicate the second harmonic violin modes.

only take lock segments with durations of more than $300 \mathrm{~s}$ into account, the duty cycle of the unperturbed data remains above $90 \%$ [2]. The longest stretch of uninterrupted lock obtained during S4 was more than $52 \mathrm{~h}$.

\section{After S4}

The next step towards completion of GEO600 was exchanging the previous MPR for one with a transmission of $900 \mathrm{ppm}$. This increased the PR factor from 300 to about 1000 giving a PR power up to about $2 \mathrm{~kW}$. The expected PR factor assuming intra-cavity losses of about $350 \mathrm{ppm}$ is slightly higher (1400). The measured cavity pole of $15 \mathrm{~Hz}$, on the other hand, agrees with the expected value. These results (especially the unexpectedly high power loss inside the PR cavity) are still inconclusive and are subject to further investigations.

\subsection{Thermal lensing of the beam splitter substrate}

The increased PR power in combination with some residual absorption leads to a thermal lens inside the BS substrate, which deforms the transmitted wave front and results in a decreased Michelson contrast. Preliminary investigations showed that the resulting thermal lens can be explained by absorption losses of less than $0.5 \mathrm{ppm} \mathrm{cm}^{-1}$ in the BS which holds promising prospects for future power increases.

\subsection{Digital SR control loop}

In order to ease changes in and optimization of the longitudinal SR servo transfer function we switched from analogue to digital electronics using a dSPACE ${ }^{\mathrm{TM}}$ digital processing system. 


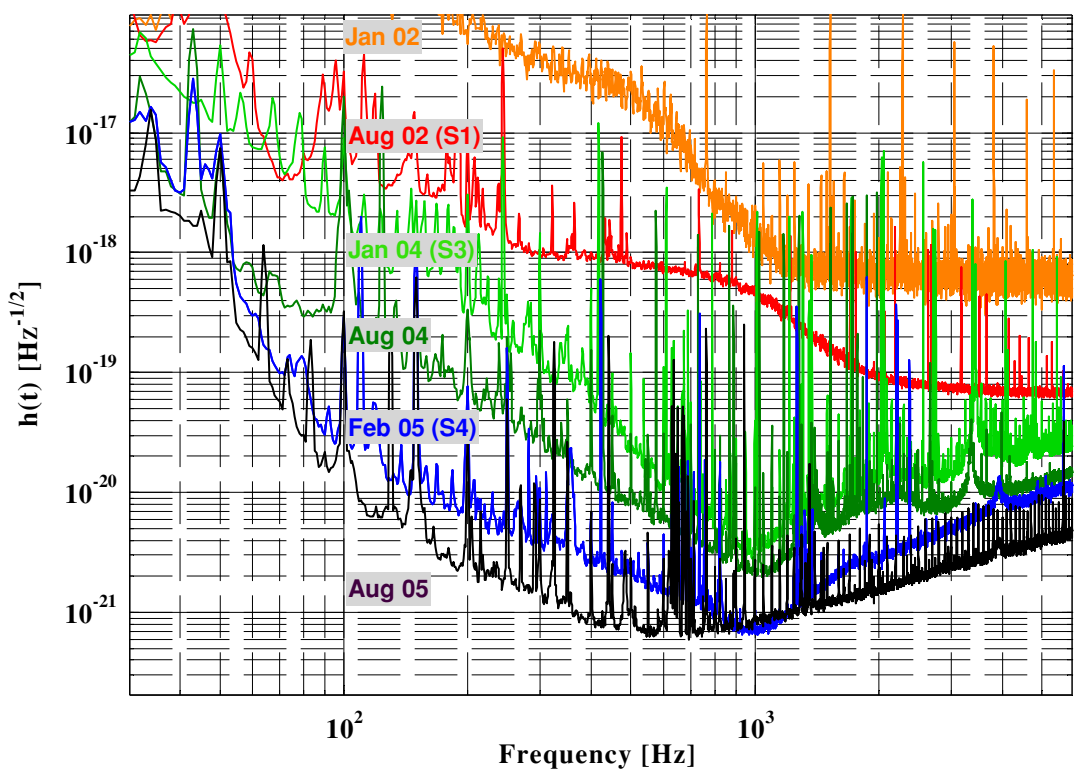

Figure 5. Sensitivity development of GEO600. The tuning of the SR cavity is $1 \mathrm{kHz}$ for the 5 earlier times and $300 \mathrm{~Hz}$ for the August 2005 graph. Almost all of the lines in the August 2005 sensitivity graph are calibration lines, violin modes or harmonics of $50 \mathrm{~Hz}$.

This allowed the use of complex filter functions with high gain at low frequencies and a steep roll-off above unity gain frequency which can be set between $20 \mathrm{~Hz}$ and $40 \mathrm{~Hz}$. Their use improved the sensitivity from $150 \mathrm{~Hz}$ to $400 \mathrm{~Hz}$ by about a factor of 2 .

In order to increase the catching range for the SR mirror during lock acquisition, the SR cavity is initially tuned to a frequency of about $2.5 \mathrm{kHz}$. After lock acquisition, the SR resonance is then shifted to the desired resonance frequency with the help of a computer script that sets all the required parameters to keep the various longitudinal and auto-alignment servo loops stable and change the position of MSR. Numerical simulations gave a new set of parameters that allowed extending the tuning range from about $600 \mathrm{~Hz}$ (which was the lower limit up to S4) to $200 \mathrm{~Hz}$. Now the interferometer is routinely operated at a resonance frequency of $350 \mathrm{~Hz}$ which causes a good part of the difference between the S4 sensitivity curve and the latest one (August 2005) included in figure 5. The gap still left between the design sensitivity curve shown in figure 1 and the current sensitivity has various origins. In the frequency range above $500 \mathrm{~Hz}$ shot noise from the reduced laser power and the missing power build-up in combination with photo-detector noise leads to the observed sensitivity. At lower frequencies noise arising in the feedback electronics, alignment noise, scattered light and some other still unknown sources reduce the sensitivity. The injected power is currently being increased and we expect to remove the excess noise sources until Spring of 2006.

\section{References}

[1] Willke B et al 2004 Status of GEO600 Class. Quantum Grav. 21 S417-23

[2] Hild S et al 2006 Towards gravitational wave astronomy: commissioning and characterization of GEO600 J. Phys.: Conf. Ser. 3266

[3] Meers B J 1988 Recycling in laser-interferometric gravitational-wave detectors Phys. Rev. D 382317

[4] Zawischa I et al 2002 The GEO600 laser system Class. Quantum Grav. 19 1775-81 
[5] Plissi et al 2000 GEO600 triple pendulum suspension system: seismic isolation and control Rev. Sci. Instrum. $712539-45$

[6] Goßler S et al 2004 Damping and tuning of the fibre violin modes in monolithic silica suspensions Class. Quantum Grav. 21 S923-33

[7] Grote $\mathrm{H}$ et al 2004 Alignment control of GEO 600 Class. Quantum Grav. 21 S441-9

[8] Hewitson M et al 2004 Principles of the dual-recycled GEO600 Rev. Sci. Instrum. 754702

Hewitson M et al 2004 Calibration of the dual-recycled GEO600 detector for the S3 science run Class. Quantum Grav. 21 S1711-22

[9] Hewitson M et al 2005 Optimal time-domain combination of the two calibrated output quadratures of GEO600 Class. Quantum Grav. 224253

[10] Smith J R 2004 Commissioning characterization and operation of the dual-recycled GEO600 Class. Quantum Grav. 21 S1737-45 